\title{
LOCALLY CONVEX HYPERSURFACES OF CONSTANT CURVATURE WITH BOUNDARY
}

\author{
BO GUAN AND JOEL SPRUCK
}

\section{INTRODUCTION}

Given a smooth symmetric function $f$ of $n(n \geq 2)$ variables and a disjoint collection $\Gamma=\left\{\Gamma_{1}, \ldots, \Gamma_{m}\right\}$ of closed smooth embedded $(n-1)$ dimensional submanifolds of $\mathbb{R}^{n+1}$, it is a fundamental question in classical differential geometry to decide whether there exist (immersed) hypersurfaces $M$ in $\mathbb{R}^{n+1}$ of constant curvature

$$
f(\kappa[M])=K
$$

with boundary

$$
\partial M=\Gamma
$$

for some constant $K$, where $\kappa[M]=\left(\kappa_{1}, \ldots, \kappa_{n}\right)$ denotes the principal curvatures of $M$. Important examples include the classical Plateau problem for minimal or constant mean curvature surfaces and the corresponding problem for Gauss curvature, which was treated recently by the authors [12] and independently by Trudinger-Wang [24]. In this paper as in our previous work [12], we are concerned with locally convex hypersurfaces. Accordingly, the function $f$ is assumed to be defined in the convex cone $\Gamma_{n}^{+} \equiv\left\{\lambda \in \mathbb{R}^{n}\right.$ : each component $\left.\lambda_{i}>0\right\}$ in $\mathbb{R}^{n}$ and satisfy the fundamental structure conditions:

$$
f_{i}(\lambda) \equiv \frac{\partial f(\lambda)}{\partial \lambda_{i}}>0 \text { in } \Gamma_{n}^{+}, \quad 1 \leq i \leq n
$$

and

$$
f \text { is a concave function. }
$$

In addition, $f$ will be assumed to satisfy some more technical assumptions. These include

$$
f>0 \text { in } \Gamma_{n}^{+}, f=0 \text { on } \partial \Gamma_{n}^{+},
$$

Research of both authors was partially supported by NSF grants. 


$$
\sum f_{i}(\lambda) \lambda_{i} \geq \sigma_{0} \text { on }\left\{\lambda \in \Gamma_{n}^{+}: \psi_{0} \leq f(\lambda) \leq \psi_{1}\right\}
$$

for any $\psi_{1}>\psi_{0}>0$, where $\sigma_{0}$ is a positive constant depending on $\psi_{0}, \psi_{1}$, and for every $C>0$ and every compact set $E$ in $\Gamma_{n}^{+}$there exists $R=R(E, C)>0$ such that

$$
f\left(\lambda_{1}, \cdots, \lambda_{n-1}, \lambda_{n}+R\right) \geq C, \quad \forall \lambda \in E,
$$

and

$$
f(R \lambda) \geq C, \quad \forall \lambda \in E .
$$

This allows a large family $f=\sum f_{l}$ where each $f_{l}$ is of the form

$$
f_{l}=S_{n}^{\frac{1}{n N_{l}}} \prod_{i=1}^{N_{l}-1}\left(c_{i}+\sum_{k=1}^{n-1} c_{i, k} S_{n, k}^{\frac{1}{n-k}}\right)^{\frac{1}{N_{l}}}
$$

where $c_{i}, c_{i, k} \geq 0$ are constant, $c_{i}+\sum_{k} c_{i, k}>0$ for each $i, S_{k}$ is the $k$ th elementary symmetric function $\left(S_{0}=1\right)$ and $S_{k, l}=S_{k} / S_{l}(0 \leq l<k \leq n)$. However the pure curvature quotient $S_{n, k}^{\frac{1}{n-k}}$ does not satisfy (1.7).

Our first main result of this article may be stated as follows.

Theorem 1.1. Assume there exists a connected locally convex immersed hypersurface $\Sigma$ with $\partial \Sigma=\Gamma$, and $\Sigma$ is $C^{2}$ and locally strictly convex along its boundary. Let $\mathfrak{A}[\Sigma]$ be the collection of $\Sigma$-admissible (see section 3) locally convex hypersurfaces.

a) The number of homeomorphic classes in $\mathfrak{A}[\Sigma]$ is finite.

b) Let $\mathfrak{H}$ be a homeomorphic class in $\mathfrak{A}[\Sigma]$ and $\mathfrak{H}_{K}=\{M \in \mathfrak{H}: f(\kappa[M]) \geq K\}$ where $K \leq \min _{\Sigma} f(\kappa[\Sigma])$ is a nonnegative constant. Suppose $f$ satisfies the structure conditions (1.3)-(1.7). Then there exists a hypersurface $M$ in $\mathfrak{H}_{K}$ satisfying (1.1) provided that $\mathfrak{H}_{K} \neq \emptyset$.

c) Let $M \in \mathfrak{A}[\Sigma]$ satisfy (1.1). Under the conditions (1.3)-(1.8), $M$ is smooth (up to the boundary) and locally strictly convex if $K>0$. If $K=0, M$ is $C^{1,1}$ up to the boundary.

It is necessary in Theorem 1.1 to assume $\Sigma$ to be locally strictly convex along its boundary. The assumption is natural geometrically in view of the fact that there are topological obstructions to the existence of locally strictly convex hypersurfaces spanning a given $\Gamma$ (see [19]). On the other hand, it is an interesting question whether the number of homeomorphic classes of locally convex hypersurfaces spanning a given $\Gamma$ is always finite. 
Since $[\Sigma]_{K} \neq \emptyset$ (where $[\Sigma]$ denotes the homeomorphic class of $\Sigma$ in $\mathfrak{A}[\Sigma]$ ), we have in particular

Corollary 1.2. Under the assumptions of Theorem 1.1 there exists a locally convex hypersurface $M$ homeomorphic to $\Sigma$ satisfying (1.1) and (1.2). Moreover $M$ is smooth and locally strictly convex up to the boundary if $K>0$, and $M$ is $C^{1,1}$ (up to the boundary) when $K=0$.

Theorem 1.1 and Corollary 1.2 extend the results of our paper [12] which considered only the Gauss Kronecker curvature $f=S_{n}$. As in [12] we will use the Perron method to prove Theorem $1.1 \mathrm{~b}$ ) and Corollary 1.2; the resulting hypersurface will be referred as the Perron solution. This method is based on the solvability of the problem in the non-parametric setting (the Dirichlet problem) and an important uniform local graph representation property of hypersurfaces in $\mathfrak{A}[\Sigma]$ (Theorem 3.1) which was first proved in [12], and independently in [24] in somewhat less generality.

The second main result of this article introduces a variational type approach to the problem, which has the potential to be extended to more general situations. The critical observation to this approach is that every volume minimizer in $\mathfrak{H}_{K}$ in fact satisfies (1.1). The problem is thus reduced to the existence of volume minimizers in $\mathfrak{H}_{K} \cdot$

Theorem 1.3. Under the assumptions of Theorem $1.1 \mathrm{~b}$ ), every volume minimizer $M$ in $\mathfrak{H}_{K}$ satisfies (1.1). If in addition $f$ satisfies

$$
\sum f_{i}(\lambda) \lambda_{i}^{2} \geq \sigma_{2} \text { on }\left\{\lambda \in \Gamma_{n}^{+}: \psi_{0} \leq f(\lambda) \leq \psi_{1}\right\}
$$

for any $\psi_{1}>\psi_{0}>0$, where $\sigma_{2}=\sigma_{2}\left(\psi_{0}, \psi_{1}\right)$ is a positive constant depending on $\psi_{0}, \psi_{1}$, then there exists a volume minimizer $M$ in $\mathfrak{H}_{K}$, i.e.

$$
\operatorname{Vol}(M)=\min _{N \in \mathfrak{H}_{K}} \operatorname{Vol}(N),
$$

provided that $\mathfrak{H}_{K} \neq \emptyset$.

By Theorem $1.1 \mathrm{c}$ ) volume minimizers in $\mathfrak{H}_{K}$ are smooth and locally strictly convex for $K>0$. The first part of Theorem 1.3 actually holds for much more general classes of hypersurfaces (not necessarily locally convex). But to maintain the focus of this article, we will not go into detail about it here. We remark that the volume minimizer in $\mathfrak{H}_{K}$ in general can be different from the Perron solution. Whether or not the volume minimizer in each $\mathfrak{H}_{K}$ is unique seems to be an interesting question. 
In order to prove Theorems 1.1 and 1.3 we need to study the corresponding Dirichlet problem

$$
\begin{aligned}
f(\kappa[u]) & =\psi(x, u) \text { in } \Omega \\
u & =\varphi \text { on } \partial \Omega
\end{aligned}
$$

where $\kappa[u]$ denotes the principal curvatures of the graph of $u$.

Theorem 1.4. Let $\Omega$ be a bounded smooth domain in $\mathbb{R}^{n}$ and $\psi \in C^{\infty}(\bar{\Omega} \times \mathbb{R}), \psi>0$. Assume there exists a locally convex viscosity subsolution $\underline{u} \in C^{0,1}(\bar{\Omega})$ of (1.11), i.e.,

$$
\begin{aligned}
f(\kappa[\underline{u}]) & \geq \psi(x, \underline{u}) \text { in } \Omega \\
u & =\varphi \text { on } \partial \Omega,
\end{aligned}
$$

and $\underline{u}$ is $C^{2}$ and locally strictly convex (up to the boundary) in a neighborhood of $\partial \Omega$. Suppose $f$ satisfies the structure conditions (1.3)-(1.7). Then there exists a locally strictly convex solution $u \in C^{\infty}(\bar{\Omega})$ of (1.11) satisfying $u \geq \underline{u}$ on $\bar{\Omega}$. Moreover, the solution is unique if $\psi_{u} \geq 0$.

The Dirichlet problem (1.11) was first studied by Caffarelli-Nirenberg-Spruck [5] under essentially the same assumptions as ours for functions $f$ defined on larger convex cones in $\mathbb{R}^{n}$ (so the solutions are not necessarily locally convex) but only for convex domains and constant boundary data, and by Trudinger [22] for viscosity solutions. Ivochkina, Lin and Trudinger ([13], [14], [18], [15]) treated the cases of elementary symmetric functions $f=S_{k}^{\frac{1}{k}}$ and their quotients $f=S_{k, l}^{\frac{1}{k-l}}(1 \leq l<k \leq n)$ for $(k-1)$ convex domains. Sheng, Ubras and Wang recently derived a Pogorelov type interior curvature estimate for solutions of (1.11) in [20] where they also proved Corollary 1.2 for $f=S_{n, k}^{\frac{1}{n-k}}$ (and $K>0$ ), extending a result of Ivochkina and Tomi [16]. As in our earlier work [11], [7], we emphasize in Theorem 1.4 the importance of allowing domains of arbitrary geometry, assuming the existence of a subsolution achieving the boundary data. We hope to extend Theorem 1.4 to larger classes of curvature functions in future work.

This article is organized as follows. In section 2 where the Dirichlet problem is treated, we derive a priori boundary estimates for second derivatives in order to prove Theorem 1.4. In section 3 we prove the existence of Perron solutions and volume minimizers in $\mathfrak{H}_{K}$ when it is nonempty, and complete the proofs of Theorem $1.1 \mathrm{a}$ ), b) and Theorem 1.3. Finally the regularity and local strict convexity of solutions to (1.1) in $\mathfrak{A}[\Sigma]$ (Theorem $1.1 \mathrm{c})$ ) is proved in section 4. 
Part of this work was done while the first author was visiting the Department of Mathematics at Johns Hopkins University and he gratefully acknowledges their hospitality.

\section{The Dirichlet PRoblem: Boundary estimates for SECOND DERIVATIVES}

The primary purpose of this section is to prove Theorem 1.4. This is based on the establishment of the a priori $C^{2}$ estimates for locally convex solutions.

Theorem 2.1. Let $u \in C^{\infty}(\bar{\Omega})$ be a locally convex solution of (1.11) satisfying $u \geq \underline{u}$ in $\Omega$. Then

$$
|u|_{C^{2}(\bar{\Omega})} \leq C
$$

A bound for the $C^{1}$ norm $|u|_{C^{1}(\bar{\Omega})}$ follows from the convexity of $u$ and the inequality $u \geq \underline{u}$ in $\bar{\Omega}$. In [5], it is shown how to derive the global estimates for $\left|D^{2} u\right|$ on $\bar{\Omega}$ from its bound on the boundary $\partial \Omega$. We therefore only have to establish the boundary estimate

$$
\left|D^{2} u\right| \leq C \text { on } \partial \Omega \text {. }
$$

Before we proceed to proving (2.2) let us recall from [4] a reformulation of equation (1.1) in the form

$$
G\left(D^{2} u, D u\right)=\psi(x, u) .
$$

For the graph of $u$ the induced metric, its inverse matrix and second fundamental form are given respectively by

$$
\begin{gathered}
g_{i j}=\delta_{i j}+u_{i} u_{j}, \\
g^{i j}=\delta_{i j}-\frac{u_{i} u_{j}}{w^{2}}, \quad w=\sqrt{1+|D u|^{2}},
\end{gathered}
$$

and

$$
h_{i j}=\frac{u_{i j}}{w} .
$$

Following [4], the principal curvatures of graph $u$ are the eigenvalues of the symmetric matrix $A[u]=\left[a_{i j}\right]$ :

$$
a_{i j}=\frac{1}{w} \gamma^{i k} u_{k l} \gamma^{l j}
$$


where $\left[\gamma^{i k}\right]$ and its inverse matrix $\left[\gamma_{i k}\right]$ are given respectively by

$$
\gamma^{i k}=\delta_{i k}-\frac{u_{i} u_{k}}{w(1+w)}
$$

and

$$
\gamma_{i k}=\delta_{i k}+\frac{u_{i} u_{k}}{1+w}
$$

Geometrically, $\left[\gamma_{i k}\right]$ is the square root of the metric, i.e., $\gamma_{i k} \gamma_{k j}=g_{i j}$.

Let $\mathfrak{S}$ be the set of $n \times n$ symmetric matrices and $\mathfrak{S}^{+}=\{A \in \mathfrak{S}: A>0\}$, i.e. the set of positive definite symmetric matrices. Introducing the function $F$ defined by

$$
F(A)=f(\lambda(A)), \quad A \in \mathfrak{S}^{+}
$$

where $\lambda(A)$ denotes the eigenvalues of $A$, equation (1.11) thus can be written in the form

$$
F(A[u])=\psi(x, u) .
$$

Therefore, the function $G$ in (2.3) is defined by

$$
G(r, p):=F(A(r, p)), \quad r \in \mathfrak{S}^{+}, p \in \mathbb{R}^{n}
$$

where $A(r, p)$ is obtained from $A[u]$ with $(r, p)$ in place of $\left(D^{2} u, D u\right)$.

We next recall some properties of the functions $F$ and $G$. We will use the notation

$$
F^{i j}(A)=\frac{\partial F}{\partial a_{i j}}(A), \quad F^{i j, k l}(A)=\frac{\partial^{2} F}{\partial a_{i j} \partial a_{k l}}(A) .
$$

The matrix $\left[F^{i j}(A)\right]$ is symmetric and has eigenvalues $f_{1}, \ldots, f_{n}$. By assumption (1.3), $\left[F^{i j}(A)\right]$ is therefore is positive definite for $A \in \mathfrak{S}^{+}$, while (1.4) implies that $F$ is a concave function of $A \in \mathfrak{S}^{+}$(see [3]), that is

$$
F^{i j, k l}(A) \xi_{i j} \xi_{k l} \leq 0, \quad \forall\left[\xi_{i j}\right] \in \mathfrak{S}, A \in \mathfrak{S}^{+} .
$$

If $P$ is a nondegenerate matrix, by the identity

$$
F\left(P A P^{-1}\right)=F(A)
$$

we see that

$$
P\left[F^{i j}(A)\right] P^{-1}=\left[F^{i j}\left(P A P^{-1}\right)\right] .
$$

It follows that $\left[F^{i j}(A)\right]$ and $A$ can be diagonalized simultaneously by an orthonormal transformation. Consequently, the eigenvalues of the matrix $\left[F^{i j}(A)\right] A$, which is not 
necessarily symmetric, and those of $A\left[F^{i j}(A)\right] A$ are given by

$$
\lambda\left(\left[F^{i j}(A)\right] A\right)=\left(f_{1} \lambda_{1}, \ldots, f_{n} \lambda_{n}\right)
$$

and, respectively,

$$
\lambda\left(A\left[F^{i j}(A)\right] A\right)=\left(f_{1} \lambda_{1}^{2}, \ldots, f_{n} \lambda_{n}^{2}\right) .
$$

In particular,

$$
\begin{gathered}
F^{i j}(A) a_{i j}=\sum f_{i} \lambda_{i}, \\
F^{i j}(A) a_{i k} a_{j k}=\sum f_{i} \lambda_{i}^{2} .
\end{gathered}
$$

Equation (2.3) satisfies similar structure conditions to those of (2.5). We have

$$
G^{i j}:=\frac{\partial G}{\partial r_{i j}}=\frac{1}{w} F^{k l} \gamma^{i k} \gamma^{l j} .
$$

So equation (2.3) is elliptic for locally strictly convex solutions. Moreover, $G(r, p)$ is a concave function of $r \in \mathfrak{S}^{+}$when condition (1.4) holds. We also have

$$
\begin{gathered}
\frac{1}{w^{3}} \sum F^{i i} \leq \sum G^{i i} \leq \frac{1}{w} \sum F^{i i}, \\
G^{i j} u_{i j}=\sum f_{i} \kappa_{i},
\end{gathered}
$$

and

$$
G^{i j} g^{k l} u_{i l} u_{k j}=w \sum f_{i} \kappa_{i}^{2} .
$$

It is obvious that under condition (1.8) $G$ satisfies

$$
\lim _{R \rightarrow+\infty} G(R r, p)=+\infty, \forall p, \in \mathbb{R}^{n}, \forall r \in \mathfrak{S}^{+} .
$$

Similarly, we have

Lemma 2.2. If $f$ satisfies (1.7) then

$$
\lim _{R \rightarrow+\infty} G(r+R q \otimes q, p)=+\infty, \quad \forall p, q \in \mathbb{R}^{n}, q \neq 0, \forall r \in \mathfrak{S}^{+} .
$$

Proof. Without loss of generality we may assume $q=(0, \ldots, 0,1)$. Writing $A(r, p)=$ $\left[a_{i j}\right]$ and $A(r+R q \otimes q, p)=\left[\tilde{a}_{i j}\right]$, we then have

$$
\tilde{a}_{i j}=a_{i j}+R \gamma^{i n} \gamma^{n j}
$$

where

$$
\gamma^{i k}=\gamma^{i k}(p):=\delta_{i k}-\frac{p_{i} p_{k}}{\sqrt{1+|p|^{2}}\left(1+\sqrt{1+|p|^{2}}\right)}
$$


The eigenvalues of $\left[\gamma^{i k}\right]$ are 1 of multiplicity $n-1$ and $1 / \sqrt{1+|p|^{2}}$ of multiplicity 1 . Therefore, after an orthonormal transformation we may assume

$$
\tilde{a}_{i j}=a_{i j}+\left(1+|p|^{2}\right)^{-\frac{1}{2}} R \delta_{i n} \delta_{n j} .
$$

By Lemma 1.2 of [3] the eigenvalues of $A(r+R q \otimes q, p)$ are given by

$$
\lambda_{\alpha}=\lambda_{\alpha}^{\prime}+o(1), \alpha \leq n-1 ; \quad \lambda_{n}=R+O(1)
$$

as $R$ tends to infinity, where $\lambda_{\alpha}^{\prime}, 1 \leq \alpha<n$ are the eigenvalues of $\left[a_{\alpha \beta}\right]_{1 \leq \alpha, \beta<n}$. Since $\left(\lambda_{1}^{\prime}, \ldots, \lambda_{n-1}^{\prime}, 1\right) \in \Gamma_{n}^{+},\left(\lambda_{1}, \ldots, \lambda_{n-1}, 1\right)$ belongs to a compact subset of $\Gamma_{n}^{+}$for all $R$ sufficiently large. Consequently, (2.15) follows from (1.7).

In order to derive (2.2) we need some special properties of the linearized operator

$$
L=G^{i j} \partial_{i} \partial_{j}+G^{i} \partial_{i}
$$

where $G^{i j}=G^{i j}\left(D^{2} u, D u\right), G^{i}=\frac{\partial G}{\partial p_{i}}\left(D^{2} u, D u\right)$. First, we observe that

Lemma 2.3. For some constant $C_{0}>0$

$$
\sum\left|G^{i}\right| \leq C_{0} \sum f_{i}\left|\kappa_{i}\right|
$$

In particular, $\sum\left|G^{i}\right|$ is bounded if $u$ is locally convex.

Proof. This follows from some straightforward calculations. For the reader's convenience we include an outline here. First,

$$
\begin{aligned}
G^{s} & =F^{i j} u_{k l} \frac{\partial}{\partial u_{s}}\left(\frac{1}{w} \gamma^{i k} \gamma^{l j}\right) \\
& =-\frac{u_{s}}{w^{2}} F^{i j} a_{i j}-\frac{2}{w} F^{i j} \gamma^{i k} u_{k l} \frac{\partial \gamma^{l j}}{\partial u_{s}} .
\end{aligned}
$$

¿From (2.4) we have

$$
\gamma^{i k} u_{k l}=w a_{i k} \gamma_{k l}
$$

It follows that

since $\gamma_{k l} \gamma^{l j}=\delta_{k j}$. Next,

$$
\gamma^{i k} u_{k l} \frac{\partial \gamma^{l j}}{\partial u_{s}}=w a_{i k} \gamma_{k l} \frac{\partial \gamma^{l j}}{\partial u_{s}}=-w a_{i k} \gamma^{l j} \frac{\partial \gamma_{k l}}{\partial u_{s}}
$$

$$
\frac{\partial \gamma_{k l}}{\partial u_{s}}=\frac{u_{k} \delta_{l s}+u_{l} \gamma^{k s}}{1+w}
$$

and

$$
u_{l} \gamma^{l j}=\frac{u_{j}}{w} .
$$


Thus

¿From (2.18) and (2.6) we obtain

$$
\gamma^{i k} u_{k l} \frac{\partial \gamma^{l j}}{\partial u_{s}}=\frac{a_{i k}\left(w u_{k} \gamma^{s j}+u_{j} \gamma^{k s}\right)}{1+w}
$$

$$
G^{s}=-\frac{u_{s}}{w^{2}} \sum f_{i} \kappa_{i}-\frac{2}{w(1+w)} F^{i j} a_{i k}\left(w u_{k} \gamma^{s j}+u_{j} \gamma^{k s}\right)
$$

and therefore (2.17). In particular, if $u$ is locally convex then $\kappa_{i} \geq 0$ and, consequently,

$$
\sum\left|G^{i}\right| \leq C_{0} \sum f_{i} \kappa_{i} \leq C_{0} f(\kappa[u])
$$

by the concavity of $f$ and the assumption $f(0)=0$.

It is sometimes convenient to use the following expression for $G^{s}$ :

$$
G^{s}=-\frac{u_{s}}{w^{2}} \sum f_{i} \kappa_{i}-\frac{2}{w(1+w)} G^{i j} u_{i k}\left(w u_{j} \gamma^{k s}+u_{k} \gamma^{j s}\right) .
$$

This follows from (2.18) combined with (2.10), (2.19), (2.20) and

$$
\frac{\partial \gamma^{l j}}{\partial u_{s}}=-\gamma^{i j} \gamma^{l k} \frac{\partial \gamma_{i k}}{\partial u_{s}}
$$

By (2.12), (2.23) and (2.20) we obtain

$$
L u=\frac{1}{w^{2}} \sum f_{i} \kappa_{i}-\frac{2}{w} G^{i j} w_{i} u_{j} .
$$

For later reference we also record the following identity

$$
L w=\frac{u_{k} L u_{k}}{w}+\sum f_{i} \kappa_{i}^{2}
$$

which follows from (2.13) and, by a straightforward calculation

$$
w_{i}=\frac{u_{k} u_{k i}}{w}, \quad w_{i j}=\frac{u_{k} u_{k i j}}{w}+\frac{1}{w} g^{k l} u_{k i} u_{l j}
$$

The following lemma will be the key ingredient in the proof of (2.2). It would be interesting to know if it still holds without assumption (1.7).

Lemma 2.4. Assume $f$ satisfies (1.3)-(1.7). For any constant $C_{0}>0$, there exist positive constants $t, \delta$ sufficiently small and $N$ sufficiently large such that the function $v=u-\underline{u}+t d-N d^{2}$ satisfies

$$
\begin{cases}L v \leq-C_{0}-\beta \sum G^{i i} & \text { in } \Omega \cap B_{\delta}, \\ v \geq 0 & \text { on } \partial\left(\Omega \cap B_{\delta}\right),\end{cases}
$$

where $\beta>0$ depends only on the convexity of $\underline{u}, d$ is the distance function to $\partial \Omega$, and $B_{\delta}$ is a ball of radius $\delta$ centered at a point on $\partial \Omega$. 
Proof. We first note that, since $\underline{u}$ is $C^{2}$ and locally strictly convex in a neighborhood of $\partial \Omega$, we have

$$
D^{2} \underline{u} \geq 4 \beta I \text { in } \bar{\Omega} \cap B_{\delta}
$$

for some fixed $\beta>0$ when $\delta$ is small enough. Thus $\lambda\left(D^{2} \underline{u}-3 \beta I\right)$ lies in a compact set of $\Gamma_{n}^{+}$. Since $|D d|=1$ and $-C I \leq D^{2} d \leq C I$ where $C$ only depends on $\delta$ and the geometric quantities of $\partial \Omega$, we have

$$
L d \leq C \sum G^{i i}+\sum\left|G^{i}\right| \text { in } \Omega \cap B_{\delta}
$$

and

$$
\lambda\left(D^{2}\left(\underline{u}+N d^{2}\right)-2 \beta I\right) \geq \lambda\left(D^{2} \underline{u}-3 \beta I+2 N D d \otimes D d\right) \text { in } \Omega \cap B_{\delta}
$$

when $\delta$ is small (so that $2 N \delta D^{2} d \geq-\beta I$ ).

Next, it follows from the concavity of $F$ that the function $G(r, p)$ is concave with respect to $r$. Therefore

$$
\begin{gathered}
L\left(u-\underline{u}-N d^{2}\right)+2 \beta \sum G^{i i}=G^{i j}\left(u-\underline{u}-N d^{2}\right)_{i j} \\
+2 \beta \sum G^{i i}+G^{i}\left(u-\underline{u}-N d^{2}\right)_{i} \\
\leq G\left(D^{2} u, D u\right)-G\left(D^{2}\left(\underline{u}+N d^{2}\right)-2 \beta I, D u\right) \\
+G^{i}(u-\underline{u})_{i}-2 N d G^{i} d_{i} .
\end{gathered}
$$

By Lemma 2.3 we have

$$
\sum\left|G^{i}\right| \leq C
$$

where $C$ depends on $|u|_{C^{0}(\bar{\Omega})}$. Consequently, by Lemma 2.2 we may choose $N$ sufficiently large (depending on $|D u|_{C^{0}(\bar{\Omega})}$ ) such that

$$
G\left(D^{2}\left(\underline{u}+N d^{2}\right)-2 \beta I, D u\right) \geq G\left(D^{2} u, D u\right)+G^{i}(u-\underline{u})_{i}+\sum\left|G^{i}\right|+C_{0}
$$

in $\Omega \cap B_{\delta}$ when $\delta$ is sufficiently small (depending on $N$ ). It follows that

$$
\begin{aligned}
L v \leq-C_{0}- & (2 \beta-C t) \sum G^{i i} \\
& -(1-t-2 N d) \sum\left|G^{i}\right| \\
& \leq-C_{0}-\beta \sum G^{i i} \text { in } \Omega \cap B_{\delta}
\end{aligned}
$$

when $t$ and $\delta$ are sufficiently small. Finally, for fixed $t$ and $N$ we can require $\delta \leq t / N$ to ensure $v \geq 0$ on $\partial\left(\Omega \cap B_{\delta}\right)$. 
Lemma 2.5. Let $h \in C^{2}\left(\overline{\Omega \cap B_{\delta}}\right)$ where $B_{\delta}$ is centered at the origin which is on $\partial \Omega$. Suppose $h$ satisfies $h \leq C_{0}|x|^{2}$ on $(\partial \Omega) \cap B_{\delta}, h(0)=0$ and

$$
-L h \leq C_{1}\left(1+\sum G^{i i}\right) \text { in } \Omega \cap B_{\delta} .
$$

Then, under the structure conditions (1.3)-(1.6) and (1.7), $h_{n}(0) \leq C$, where $C$ depends on $\beta^{-1}, C_{0}, C_{1},|h|_{C^{0}\left(\overline{\Omega \cap B_{\delta}}\right)}$ and $|u|_{C^{1}(\bar{\Omega})}$.

Proof. By Lemma 2.4, $A v+B|x|^{2}-h \geq 0$ on $\partial\left(\Omega \cap B_{\delta}\right)$ and

$$
L\left(A v+B|x|^{2}-h\right) \leq 0 \text { in } \Omega \cap B_{\delta}
$$

when $A \gg B$ are both large. Thus $A v+B|x|^{2}-h \geq 0$ in $\overline{\Omega \cap B_{\delta}}$ by the maximum principle. Consequently,

$$
A v_{n}(0)-h_{n}(0)=D_{n}\left(A v+B|x|^{2}-h\right)(0) \geq 0
$$

since $A v+B|x|^{2}-h=0$ at the origin.

We are now ready to derive the boundary estimate (2.2).

Proof of (2.2). Consider any fixed point on $\partial \Omega$; we may assume it to be the origin of $\mathbb{R}^{n}$ and choose the coordinates so that the positive $x_{n}$ axis is the interior normal to $\partial \Omega$ at 0 . Near the origin, $\partial \Omega$ can be represented as a graph

$$
x_{n}=\rho\left(x^{\prime}\right)=\frac{1}{2} \sum_{\alpha, \beta<n} B_{\alpha \beta} x_{\alpha} x_{\beta}+O\left(\left|x^{\prime}\right|^{3}\right), \quad x^{\prime}=\left(x_{1}, \ldots, x_{n-1}\right) .
$$

In this proof we will assume that $\varphi$ has been extended to a harmonic function on the whole $\Omega$. Since $u-\varphi=0$ on $\partial \Omega$,

$$
(u-\varphi)_{\alpha \beta}(0)=-(u-\varphi)_{n}(0) B_{\alpha \beta}, \quad \alpha, \beta<n .
$$

It follows that

$$
\left|u_{\alpha \beta}(0)\right| \leq C, \quad \alpha, \beta<n .
$$

Next, for fixed $\alpha<n$ consider the operator

$$
T=\partial_{\alpha}+\sum_{\beta<n} B_{\alpha \beta}\left(x_{\beta} \partial_{n}-x_{n} \partial_{\beta}\right) .
$$

By [5] we have $L(T u)=T \psi(x, u)$. It follows that

$$
|L T(u-\varphi)| \leq C\left(1+\sum G^{i i}\right) .
$$


Note that $|T(u-\varphi)| \leq C$ in $\bar{\Omega}$. Moreover, since $u-\varphi=0$ on $\partial \Omega$, near the origin we have

$$
|T(u-\varphi)| \leq C|x|^{2} \text { on } \partial \Omega
$$

Applying Lemma 2.5 to $h= \pm T(u-\varphi)$, it follows that

$$
\left|u_{\alpha n}(0)\right| \leq C \text {. }
$$

Remark 2.6. In [11], page 611, and subsequent papers by the first author (see [7], [8]) we used $T(u-\underline{u})$ instead of $T(u-\varphi)$ in similar calculations. As a result, the constants in the corresponding inequalities to (2.30) depend on the third derivatives of $\underline{u}$. To avoid this, we should as in (2.30) replace $T(u-\underline{u})$ by $T(u-\varphi)$. The corresponding results in those papers remain valid. In 1999 ([9]) Pengfei Guan pointed out the improvement to the first author; recently Z. Blocki raised the same question. To both of them we wish to express our gratitude. Pengfei Guan and the first author also observed that in those papers it was enough to assume the subsolution to be $C^{2}$ only in a neighborhood of the boundary. This observation has been used in [12] and the current paper.

Let us come back to the proof of (2.2). We have so far proved

$$
\left|u_{\xi \eta}(x)\right| \leq C, \quad\left|u_{\xi \nu}(x)\right| \leq C, \quad \forall x \in \partial \Omega
$$

where $\xi, \eta$ denote any unit tangent vectors and $\nu$ the unit interior normal vector to $\partial \Omega$. We have to show

$$
u_{\nu \nu} \leq C \text { on } \partial \Omega
$$

We first prove

$$
M:=\min _{x \in \partial \Omega} \min _{\xi \in T_{x}(\partial \Omega),|\xi|=1} u_{i j}(x) \xi_{i} \xi_{j} \geq c_{0}
$$

for some $c_{0}>0$, where $T_{x}(\partial \Omega)$ denotes the tangent space of $\partial \Omega$ at $x \in \partial \Omega$.

Let $\sigma$ be a smooth defining function of $\Omega$, that is, $\sigma$ is defined in a neighborhood of $\Omega$ satisfying

$$
\Omega=\{\sigma<0\}, \partial \Omega=\{\sigma=0\}, \text { and }|D \sigma|=1 \text { on } \partial \Omega .
$$

Since $u-\underline{u}=0$ on $\partial \Omega$, we see that $u-\underline{u}=\eta \sigma$ for some function $\eta \leq 0$. Note that $D \sigma=-\nu$ on $\partial \Omega$ where $\nu$ is the interior unit normal to $\partial \Omega$. We have $\eta=-(u-\underline{u})_{\nu}$ 
on $\partial \Omega$ and, similarly to $(2.28)$

$$
u_{i j} \xi_{i} \xi_{j}=\underline{u}_{i j} \xi_{i} \xi_{j}-(u-\underline{u})_{\nu} \sigma_{i j} \xi_{i} \xi_{j} \text { on } \partial \Omega
$$

for any tangent vector field $\xi=\left(\xi_{1} \ldots, \xi_{n}\right)$ to $\partial \Omega$.

We may choose coordinates in $\mathbb{R}^{n}$ such that $M$ is achieved at $0 \in \partial \Omega$ with $\xi=$ $(1,0, \ldots, 0)$ and $e_{n}=\nu(0)$. Thus

$$
M=u_{11}(0)=\underline{u}_{11}(0)-(u-\underline{u})_{\nu}(0) \sigma_{11}(0) .
$$

We may assume

$$
(u-\underline{u})_{\nu}(0) \sigma_{11}(0)>\frac{1}{2} \underline{u}_{11}(0)
$$

for otherwise we are done because of the uniform (local) convexity of $\underline{u}$ on $\partial \Omega$.

Let $\zeta:=\left(\zeta_{1}, \ldots, \zeta_{n}\right)$ be defined as

$$
\begin{aligned}
& \zeta_{1}=-\sigma_{n}\left(\sigma_{1}^{2}+\sigma_{n}^{2}\right)^{-\frac{1}{2}}, \\
& \zeta_{j}=0, \quad 2 \leq j \leq n-1, \\
& \zeta_{n}=\sigma_{1}\left(\sigma_{1}^{2}+\sigma_{n}^{2}\right)^{-\frac{1}{2}} .
\end{aligned}
$$

Since $\sigma_{i j} \zeta_{i} \zeta_{j}$ is continuous and $0 \leq(u-\underline{u})_{\nu} \leq C$ on $\partial \Omega$, there exists $c_{1}>0$ and $\delta>0$ (which may be assumed the same as in Lemma 2.4) such that

$$
\sigma_{i j} \zeta_{i} \zeta_{j}(x) \geq \frac{1}{2} \sigma_{i j} \zeta_{i} \zeta_{j}(0)=\frac{\sigma_{11}(0)}{2}>\frac{\underline{u}_{11}(0)}{4(u-\underline{u})_{\nu}(0)} \geq c_{1} \text { in } \Omega \cap B_{\delta}(0) .
$$

Thus the function

$$
\Phi:=\frac{\varphi_{i j} \zeta_{i} \zeta_{j}-M}{\sigma_{i j} \zeta_{i} \zeta_{j}}
$$

is smooth and bounded in $\Omega \cap B_{\delta}(0)$. Since $\zeta(x) \in T_{x}(\partial \Omega)$ for $x \in \partial \Omega$ and $|\zeta|=1$ everywhere, we have

$$
\varphi_{i j} \zeta_{i} \zeta_{j}+(D(u-\varphi) \cdot D \sigma) \sigma_{i j} \zeta_{i} \zeta_{j}=u_{i j} \zeta_{i} \zeta_{j} \geq M \text { on } \partial \Omega \text {. }
$$

It follows that

$$
\Phi+D(u-\varphi) \cdot D \sigma \geq 0 \text { on }(\partial \Omega) \cap B_{\delta}(0),
$$

Next, we claim that

$$
L(\Phi+D(u-\varphi) \cdot D \sigma) \leq C\left(1+\sum G^{i i}\right) \text { in } \Omega \cap B_{\delta}(0) .
$$


To see this we only need to check

$$
\begin{aligned}
L(D u \cdot D \sigma) & =D \sigma \cdot L(D u)+D u \cdot L(D \sigma)+2 G^{i j} u_{s i} \sigma_{s j} \\
& \leq C\left(1+\sum G^{i i}\right)+2 F^{k l} a_{k i} \gamma_{i s} \gamma^{l j} \sigma_{s j} \\
& \leq C\left(1+\sum G^{i i}\right)+C \sum f_{i} \kappa_{i} \\
& \leq C\left(1+\sum G^{i i}\right) .
\end{aligned}
$$

Here the third step follows from (2.6) while the last by the fact that $\sum f_{i} \kappa_{i} \leq f(\kappa)$ which is a consequence of the concavity of $f$ since $f(0)=0$, as in the proof of Lemma 2.3.

Now we can apply Lemma 2.5 to $h=\Phi+D(u-\varphi) \cdot D \sigma$ to conclude that

$$
u_{n n}(0) \leq \varphi_{n n}(0)+\Phi_{n}(0) \leq C .
$$

We thus have a bound $\left|D^{2} u(0)\right| \leq C$ and consequently an upper bound for all the principal curvature of the graph of $u$ at the origin. Since $f(\kappa[u]) \geq \psi_{0}>0$ in $\bar{\Omega}$ and $f=0$ on $\partial \Gamma_{n}^{+}$, the principal curvatures at the origin admit a uniform positive lower bound. This in turn yields a positive lower bound for the eigenvalues of $D^{2} u(0)$, which implies (2.35).

Finally, by (2.35) and Lemma 1.2 of [3] there exists $R>0$ depending the bounds in (2.33) such that if $u_{\nu \nu}\left(x_{0}\right) \geq R, x_{0} \in \partial \Omega$ then the eigenvalues $\left(\lambda_{1}, \ldots, \lambda_{n}\right)$ of $D^{2} u\left(x_{0}\right)$ satisfies

$$
\frac{c_{0}}{2} \leq \lambda_{\alpha} \leq C, \alpha \leq n-1 ; \quad \lambda_{n} \geq \frac{R}{2} .
$$

This implies a bound for $u_{\nu \nu}\left(x_{0}\right)$ in view of Lemma 2.2. We therefore have established (2.34) and hence (2.2).

Remark 2.7. The tangential strict convexity estimate (2.35) on $\partial \Omega$ holds in more general situations, i.e. when $f$ only satisfies (1.3) and (1.5). This is a consequence of Proposition 1.1 in [11] which we restate for more general functions $f$ as follows.

Lemma 2.8. Suppose $f$ satisfies conditions (1.3) and (1.5). Let $v \in C^{2}(\bar{\Omega})$ be a locally strictly convex function such that

$$
\begin{aligned}
f\left(\lambda\left(D^{2} v\right)\right) & \geq \psi_{0}, v \geq \underline{u} \text { in } \bar{\Omega} \\
v & =\varphi \text { on } \partial \Omega
\end{aligned}
$$


where $\psi_{0}>0$ is a constant and $\underline{u}$ is a function which is $C^{2}$ and locally strictly convex (up to the boundary) in a neighborhood of $\partial \Omega$ (not necessarily a subsolution). Then

$$
v_{i j}(x) \xi_{i} \xi_{j} \geq c_{0}|\xi|, \quad \forall x \in \partial \Omega, \forall \xi \in T_{x}(\partial \Omega)
$$

for some uniform constant $c_{0}=c_{0}\left(\Omega, \underline{u}, \psi_{0}\right)>0$.

This was proved in [11] only for the Monge-Ampère case $\left(f=S_{n}\right)$ but the same proof there works for the general case. So we omit it here and refer the reader to [11].

To see how Lemma 2.8 applies to our situation we note that, since $u$ is locally convex,

$$
f\left(\lambda\left(D^{2} u\right)\right) \geq f(\kappa[u]) .
$$

This inequality, which will also be used in section 4 , can be seen as follows. Let $\lambda_{1} \geq \cdots \geq \lambda_{n}$ and $\kappa_{1} \geq \cdots \geq \kappa_{n}$ be the eigenvalues of $D^{2} u$ and $A[u]$, respectively. For any $\xi=\left(\xi_{1}, \ldots, \xi_{n}\right) \in \mathbb{R}^{n}$, we have

$$
u_{i j} \xi_{i} \xi_{j}=w a_{k l} \gamma_{i k} \gamma_{l j} \xi_{i} \xi_{j}=w a_{k l} \xi_{k}^{\prime} \xi_{l}^{\prime}
$$

where

$$
\xi_{i}^{\prime}=\gamma_{i k} \xi_{k}=\xi_{i}+\frac{(\xi \cdot D u) u_{i}}{1+w}
$$

Note that

$$
|\xi|^{2} \leq\left|\xi^{\prime}\right|^{2}=|\xi|^{2}+|\xi \cdot D u|^{2} \leq w^{2}|\xi|^{2}
$$

where $\xi^{\prime}=\left(\xi_{1}^{\prime}, \ldots, \xi_{n}^{\prime}\right)$. Since both $D^{2} u$ and $A[u]$ are positive semidefinite, it follows from the minimax characterization of eigenvalues that

$$
w \kappa_{k} \leq \lambda_{k} \leq w^{3} \kappa_{k}, \quad 1 \leq k \leq n .
$$

This proves (2.38) and therefore (2.35).

¿From Theorem 2.1 we can apply the $C^{2, \alpha}$ estimates of Krylov [17] and the classical Schauder theory to derive a priori bounds for higher order derivatives. The existence of the desired solution in Theorem 1.4 now follows from the standard continuity method and degree arguments as in [2]. The proof of Theorem 1.4 is thus complete.

In the next two sections we will need the following existence and regularity results for Lipschitz domains and boundary data. See also Trudinger [22] and Theorem 1.2 in $[20]$. 
Theorem 2.9. Let $\Omega$ be a bounded domain in $\mathbb{R}^{n}$ with $\partial \Omega \in C^{0,1}$. Suppose there exists a locally convex viscosity subsolution $\underline{u} \in C^{0,1}(\bar{\Omega})$ of (1.11). Then there exists a locally convex viscosity solution $u \in C^{0,1}(\bar{\Omega})$ of (1.11) satisfying $u=\underline{u}$ on $\partial \Omega$. If $\underline{u}$ is constant on $\partial \Omega$ then $u \in C^{\infty}(\Omega)$.

Proof. The existence of viscosity solution $u \in C^{0,1}(\bar{\Omega})$ follows from Theorem 1.4 by approximation. The interior smoothness for constant boundary data follows from the interior curvature estimates of Sheng-Urbas-Wang [20], the Evans-Krylov interior $C^{2, \alpha}$ estimates ([6], [17]), and the Schauder theory.

\section{The Plateau Problem: Perron solutions and volume minimizers}

In this section we will prove Theorem $1.1 \mathrm{a}$ ), b) and Theorem 1.3. The proof is based on some important properties of locally convex hypersurfaces that are proved in [12] to which the reader is referred for details. We start with some preparations.

By a hypersurface in $\mathbb{R}^{n+1}$ we mean an immersion $\Phi: \Sigma^{n} \rightarrow \mathbb{R}^{n+1}$ where $\Sigma^{n}$ is a manifold of dimension $n \geq 2$, possibly with boundary $\partial \Sigma$. Similarly, the boundary of the hypersurface means the immersion $\Phi: \partial \Sigma^{n} \rightarrow \mathbb{R}^{n+1}$. When a point $p$ on the hypersurface is considered, it should be understood as one of its preimages in $\Sigma$. We will often simply call $\Sigma$ a hypersurface in $\mathbb{R}^{n+1}$. For a subset $U$ of $\mathbb{R}^{n+1}$ and a point $p \in \Sigma, \Sigma \cap_{p} U$ will denote the intrinsic component of $\Sigma \cap U$ that contains $p$, that is, $\Sigma \cap_{p} U=\Phi\left(U_{0}\right)$ where $U_{0}$ is the component of $\Phi^{-1}(\Sigma \cap U)$ that contains the point identified to $p$ in $\Phi^{-1}(p)$. In this paper, all hypersurfaces in $\mathbb{R}^{n+1}$ we consider are assumed to be compact and orientable. If two hypersurfaces have the same boundary, their orientations are assumed to be compatible, i.e. they induce the same orientation on the boundary.

For a hypersurface $\Sigma$, we will use $\operatorname{Vol}(\Sigma)$ to denote the volume of $\Sigma$ and, at a point $p \in \Sigma$ where $\Sigma$ has a tangent hyperplane, $\nu_{\Sigma}(p)$ to denote the unit normal vector consistent with the orientation of $\Sigma$. The principal curvatures, denoted as $\kappa[\Sigma]$, of $\Sigma$ are the eigenvalues of the second fundamental form with respect to the induced metric. A hypersurface $\Sigma$ is said to be locally convex if for every point on $\Sigma$ there exists a neighborhood which can be written as a convex graph $x_{n+1}=u(x), x \in \mathbb{R}^{n}$, for a suitable coordinate system in $\mathbb{R}^{n+1}$, and the convexity of $u$ is consistent with the orientation of $\Sigma$. $\Sigma$ is locally strictly convex if it is $C^{2}$ and its principal curvatures are all positive. 
In the rest of this paper, let $\Sigma$ be a connected locally convex hypersurface in $\mathbb{R}^{n+1}$ and assume $\Sigma$ to be $C^{2}$ and locally strictly convex along its boundary. We call a locally convex hypersurface $M$ in $\mathbb{R}^{n+1}$ (not necessarily connected) $\Sigma$-admissible if $\partial M=\partial \Sigma$ (and its orientation is compatible to that of $\Sigma$ ) and $M$ locally lies on the same side as $\Sigma$ of the tangent hyperplanes of $\Sigma$ along the boundary. As in Theorem 1.1 we use $\mathfrak{A}[\Sigma]$ to denote the collection of $\Sigma$-admissible hypersurfaces.

The following theorem was first proved in [12], and independently in [24] for locally convex hypersurfaces which are locally strictly convex along the boundary. (It is stated in a different but equivalent form in [24].)

Theorem 3.1. Let $M \in \mathfrak{A}[\Sigma]$. Then at every point on $M$, locally $M$ can be represented as the graph of a convex function $u$ defined in a domain $\Omega \subset \mathbb{R}^{n}$ of a fixed lower bound in size such that

$$
|u|_{C^{0,1}(\bar{\Omega})} \leq C_{1}
$$

where $C_{1}$ depends on the second fundamental form of $\partial \Sigma$, the diameter of $M$ and the lower and upper bounds for the principal curvatures of $\Sigma$ near the boundary.

By that $\Omega$ is of a lower bound in size we mean that there exists some constant $\delta_{0}>0$ such that $\Omega$ contains a ball of radius $\delta_{0}$ or a portion of a ball of radius $\delta_{0}$ separated by a smooth hypersurface (in $\mathbb{R}^{n}$ ) with controlled geometric quantities, with the center of the ball being in $\bar{\Omega}$. In [12] Theorem 3.1 was stated for a slightly smaller class of admissible hypersurfaces but its proof works for the above more general case after some minor modifications. We therefore omit it here.

An important consequence of Theorem 3.1 is the following compactness of $\mathfrak{A}[\Sigma]$ :

Theorem 3.2. Let $\left\{\Sigma_{k}\right\}$ be a sequence in $\mathfrak{A}[\Sigma]$ of uniformly bounded extrinsic diameters. Then there exists a subsequence $\left\{\Sigma_{k_{i}}\right\}$ converging in Hausdorff metric to some $M \in \mathfrak{A}[\Sigma]$ which is homeomorphic to each $\Sigma_{k_{i}}$ for $i$ large.

This follows from Theorem 3.1 and a compactness theorem of Alexander-Ghomi [1]; see Theorem 3.4 in [12].

Proof of Theorem 1.1 a) and b). To prove part a) we first observe the following fact. Suppose $M$ is a locally convex hypersurface whose boundary $\partial M$ lies on one side of a hyperplane $P$. We may assume $P=\left\{x_{n+1}=0\right\}$ and $\partial M$ lies in $\left\{x_{n+1}<0\right\}$. Then each (intrinsic) component of $M \cap\left\{x_{n+1} \geq 0\right\}$ is a convex disk with boundary lying 
on $P$. Replacing each such component $D$ by the piece of $P$ bounded by $P \cap D$, we thus obtain a locally convex hypersurface which is homeomorphic to $M$ and has the same boundary. Now suppose that $\partial M$ is contained in the interior of a polyhedron. Repeating the above procedure finitely many times, we will obtain a locally convex hypersurface which is homeomorphic to $M$, has the same boundary as $M$, and is contained in the polyhedron. Part a) of Theorem 1.1 now follows immediately from Theorem 3.2.

We next outline the proof of part b) using the Perron method, first employed in [12] and independently in [24] for the Gauss curvature case; for more details see [12].

Let $\Sigma_{0} \in \mathfrak{H}_{K}$ where $K$ is a fixed nonnegative constant. Applying Theorem 2.9 we can replace any disk $D$ on $\Sigma$ which can be represented as a graph by a uniquely determined disk $\widetilde{D}$ with $f(\kappa[\widetilde{D}])=K$ and $\partial \widetilde{D}=\partial D$. A lifting (or $K$-lifting) of $\Sigma_{0}$ is a locally convex hypersurface obtained by repeating this procedure finitely many times. Note that every lifting $\widetilde{\Sigma}$ of $\Sigma_{0}$ still belongs to $\mathfrak{H}_{K}$, i.e. $f(\kappa[\widetilde{\Sigma}]) \geq K, \partial \widetilde{\Sigma}=\Gamma$ and $\widetilde{\Sigma}$ is homeomorphic to $\Sigma_{0}$. Moreover, we can introduce a partial order $\preceq$ between liftings of $\Sigma_{0}: \Sigma_{1} \preceq \Sigma_{2}$ if and only if $\Sigma_{2}$ is a lifting of $\Sigma_{1}$ or $\Sigma_{2}=\Sigma_{1}$.

An important fact is that the volume strictly decreases under lifting (see Lemma 4.2 of [12]). This gives us a natural way to choose a suitable sequence of liftings of $\Sigma$ which converges to a locally convex immersed hypersurface $M$ with $f(\kappa[M]) \equiv K$ and $\partial M=\Gamma$. Let $\mathfrak{L}$ be the collection of liftings of $\Sigma_{0}$ and set

$$
\mu:=\inf _{L \in \mathfrak{L}} \operatorname{Vol}(L)
$$

We may select a sequence of liftings $\Sigma_{1} \preceq \Sigma_{2} \preceq \ldots$ such that $\operatorname{Vol}\left(\Sigma_{k}\right) \rightarrow \mu$ as $k$ tends to infinity. Since all liftings of $\Sigma_{0}$ are contained in a bounded region in $\mathbb{R}^{n+1}$, by Theorem $3.2\left\{\Sigma_{k}\right\}$ has a subsequence (still denoted as $\left\{\Sigma_{k}\right\}$ ) converging in Hausdorff metric to a locally convex hypersurface $M$ which, in addition, is $C^{0,1}$ up to boundary and homeomorphic to each $\Sigma_{k}$. By Lemma 4.3 of [12] we have $\operatorname{Vol}(M)=\mu$.

By Theorem 3.1, locally (possibly after passing to a subsequence) each $\Sigma_{k}$ and $M$ can be represented as the graphs of convex functions $v_{k}$ and $v$, respectively, over a fixed domain $\Omega$ such that $v_{k}$ converges to $v$ in $C^{0,1}(\bar{\Omega})$. We consider in such a setting the Dirichlet problem

$$
f(\kappa[u])=K \text { in } \bar{\Omega}, \quad u=v_{k} \text { on } \partial \Omega .
$$


Using $v_{k}$ as a subsolution we apply Theorem 2.9 to obtain for each $k$ a unique convex solution $u_{k} \in C^{0,1}(\bar{\Omega})$ of (3.2) satisfying $u_{k} \geq v_{k}$ on $\bar{\Omega}$ and

$$
\left|u_{k}\right|_{C^{0,1}(\bar{\Omega})} \leq C \text { independent of } k \text {. }
$$

Passing to a subsequence we see $u_{k}$ converges to a convex function $u$ in $C^{0,1}(\bar{\Omega})$ satisfying $f(\kappa[u])=K$ in $\bar{\Omega}$ and $u=v$ on $\partial \Omega$.

Replacing the graph of $v_{k}$ by that of $u_{k}$ over $\Omega$ we obtain a lifting $M_{k}$ of $\Sigma_{k}$. Since $\mu \leq \operatorname{Vol}\left(M_{k}\right) \leq \operatorname{Vol}\left(\Sigma_{k}\right)$ for each $k$, by Lemma 4.3 of [12] we see that the volume of $\operatorname{graph}(u)$ is the same as that of $\operatorname{graph}(v)$. This implies $u=v$ in $\bar{\Omega}$ by Lemma 4.2 of $[12]$ and proves $f(\kappa[M]) \equiv K$.

Obviously the same idea in the above argument of can be used to prove the fact that every volume minimizer $M$ in $\mathfrak{H}_{K}$ actually satisfies $f(\kappa[M]) \equiv K$. But before proceeding to the proof of Theorem 1.3 we first state the following height estimate for hypersurfaces of constant curvature satisfying the structure condition (1.9). For $f=S_{k}(1 \leq k \leq n)$ this was proved by Rosenberg [19].

Lemma 3.3. Let $M \subset\left\{x_{n+1} \leq 0\right\}$ be a smooth hypersurface satisfying $f(\kappa[M]) \equiv K$, where $K$ is a constant, with $\partial M \subset\left\{x_{n+1}=0\right\}$. Suppose $\kappa[M]$ lies in a set where $f$ satisfies the structure conditions (1.3), (1.4) and (1.9) as well as $f(0)=0$. Then there exists a positive constant $H$ depending only on $\sigma_{2}(K, K)$ such that $M \subset\{-H \leq$ $\left.x_{n+1} \leq 0\right\}$.

Proof. Applying the Alexanderov reflection principle we may assume as in [19] that $M$ is the graph of a function $u \leq 0$ over a domain $\Omega \in \mathbb{R}^{n}$ with $u=0$ on $\partial \Omega$. Using the notation from section 2 we have by (2.25) and (2.26)

$$
\mathcal{L}\left(u+\frac{a}{w}\right)=\frac{1}{w^{2}} \sum f_{i}\left(\kappa_{i}-a \kappa_{i}^{2}\right) \leq 0 \text { in } \Omega
$$

for a sufficiently large constant $a>0$, where

$$
\mathcal{L}=L+\frac{2}{w} G^{i j} w_{i} \partial_{j}
$$

Consequently $u+\frac{a}{w} \geq 0$ by the maximum principle, and $u \geq-a$ on $\bar{\Omega}$, completing the proof.

Alternatively, one can reach the same conclusion using the linearized operator on the hypersurface and the identities from Theorem 3.4 of [21], which are implicit in [10]. The above proof of the height estimate is the only place we need assumption 
(1.9). In [21] the second author proved that if $f$ is concave and homogeneous of degree one (and normalized so that $f(1, \ldots, 1)=1$ ), then

$$
\sum f_{i} \kappa_{i}^{2} \geq(f(\kappa))^{2}
$$

holds fairly generally. In particular, see Lemma 3.7, Lemma 3.8, Proposition 3.9 in [21] which shows that $\left(S_{k, l}\right)^{1 /(k-l)}, 0 \leq l<k \leq n$ satisfies this and so do their sums and products. Hence for the purposes of the current paper, all the reasonable examples which satisfy (1.1)-(1.6) satisfy a height estimate.

Proof of Theorem 1.3. Let $M$ be a volume minimizer in $\mathfrak{H}_{K}$. For any point $p \in M$ let $N$ be a simple $K$-lifting of $M$ over a disk on $M$ containing $p$. Then $N \in \mathfrak{H}_{K}$ and $\operatorname{Vol}(N) \leq \operatorname{Vol}(M)$ by Lemma 4.2 of [12]. Thus $\operatorname{Vol}(N)=\operatorname{Vol}(M)$ since $M$ is a volume minimizer in $\mathfrak{H}_{K}$. Again by Lemma 4.2 of [12] we have $N=M$. This proves $f(\kappa[M]) \equiv K$ since $f(\kappa[N](p))=K$ and $p \in M$ is arbitrary. Note that Theorem 3.1 is not needed in this part of the proof.

To prove the existence of volume minimizers in $\mathfrak{H}_{K}$ when it is nonempty, let $\left\{\Sigma_{k}\right\}$ be a volume minimizing sequence in $\mathfrak{H}_{K}$. To apply Theorem 3.2 we need to replace $\left\{\Sigma_{k}\right\}$ by a sequence that admits a uniform bound for the diameters. As in the proof of Theorem $1.1 \mathrm{a}$ ) and with the aid of the height estimate (Lemma 3.3) this can be done in the following way.

Let us first assume $\Gamma$ lies in the lower half space $\left\{x_{n+1}<0\right\}$ and consider an arbitrary $M \in \mathfrak{H}_{K}$. Then each (intrinsic) component of $M \cap\left\{x_{n+1} \geq 0\right\}$ is a convex disk. Let $D$ be such a component. By the Perron method described above we obtain a convex disk $\widetilde{D} \subset\left\{x_{n+1} \geq 0\right\}$ satisfying $f(\kappa[\widetilde{D}])=K, \partial \widetilde{D}=\partial D$ and, moreover, $\operatorname{Vol}(\widetilde{D}) \leq \operatorname{Vol}(D)$. By Lemma 4.3 in the next section $\widetilde{D}$ is smooth in interior. Thus we can apply Lemma 3.3 to conclude that $\widetilde{D}$ is contained in $\left\{0 \leq x_{n+1} \leq H\right\}$ for some uniform positive constant $H$. If we replace each such $D$ by the corresponding $\widetilde{D}$, we will obtain a hypersurface $\widetilde{M} \in \mathfrak{H}_{K}$ having the properties that $\operatorname{Vol}(\widetilde{M}) \leq \operatorname{Vol}(M)$ and that $\widetilde{M}$ is contained in $\left\{x_{n+1} \leq H\right\}$.

Now we fix a polyhedron such that $\Gamma$ is contained in its interior. For each $\Sigma_{k}$ we may repeat the above procedure (with respect to the faces of the polyhedron) finitely many times to obtain a hypersurface $\widetilde{\Sigma}_{k} \in \mathfrak{H}_{K}$ contained in a fix bounded region in $\mathbb{R}^{n+1}$ with $\operatorname{Vol}\left(\widetilde{\Sigma}_{k}\right) \leq \operatorname{Vol}\left(\Sigma_{k}\right)$.

So we may simply assume all $\Sigma_{k}$ to be contained in a fixed bounded region in $\mathbb{R}^{n+1}$ and apply Theorem 3.2. There thus exists a subsequence, which will still be denoted 
as $\Sigma_{k}$, converging in Hausdorff metric to some $M \in \mathfrak{H}$. By Lemma 4.3 of [12], $M$ satisfies (1.10).

By Theorem 3.1, near a fixed point $p \in M$ locally (possibly after passing to a subsequence) each $\Sigma_{k}$ and $M$ can be represented as the graphs of convex functions $v_{k}$ and $v$, respectively, over a fixed domain $\Omega$ such that $v_{k}$ converges to $v$ in $C^{0,1}(\bar{\Omega})$. Consequently, $v$ satisfies

$$
f(\kappa[v]) \geq K \text { in } \bar{\Omega} .
$$

This proves that $f(\kappa[M]) \geq K$ everywhere, i.e. $M \in \mathfrak{H}_{K}$ and therefore is a volume minimizer in $\mathfrak{H}_{K}$.

\section{LOCAL STRICT CONVEXITY AND SMOOTHNESS}

In this section we prove part c) of Theorem 1.1. To this end let $M \in \mathfrak{A}[\Sigma]$ satisfy $f(\kappa[M])=K$. The case $K=0$ has been proved in [12] as by assumption (1.5) the Gauss curvature of $M$ vanishes when $f(\kappa[M])=0$. In the rest of this section we thus will assume $K>0$. Note that $M$ is locally Lipschitz up to boundary so it has local supporting hyperplanes everywhere.

Lemma 4.1. Let $P$ be a supporting hyperplane to $M$ at an interior point $q \in M$. Then

$$
U_{t} \cap \partial M=\emptyset \text { for all } t>0 \text { sufficiently small }
$$

where $U_{t}=M \cap_{q}\left\{z \in \mathbb{R}^{n+1}:(z-q) \cdot \nu_{P} \leq t\right\}$. Consequently, $U_{t}$ is a convex cap for all $t>0$ small.

The following proof is a modification of an argument in [12] for the Gauss curvature case.

Proof. Suppose this is not the case. Then we can find two points $q_{1}, q_{2} \in \partial M$ such that the segment $\overline{q_{1} q_{2}} \subseteq M \cap_{q} P$ and $P$ is a local supporting plane of $M$ at every point on $\overline{q_{1} q_{2}}$. By the proof (step 3) of Theorem 3.1 of [12] $\overline{q_{1} q_{2}}$ is transversal to $\partial M$ at the endpoints. Without loss of generality we may assume $P=\left\{x_{n+1}=0\right\}$ and

$$
q_{i}=\left(0, \ldots, 0,(-1)^{i} a, 0\right), \quad i=1,2,
$$


where $a>0$. Consequently, there exists a constant $\delta>0$ such that, in a neighborhood of $\overline{q_{1} q_{2}}, M$ is given as a convex graph $x_{n+1}=u(x) \geq 0$ over a domain

$$
\Omega:=\left\{x:=\left(x^{\prime}, x_{n}\right) \in \mathbb{R}^{n} \mid \rho_{1}\left(x^{\prime}\right)<x_{n}<\rho_{2}\left(x^{\prime}\right) \text { for }\left|x^{\prime}\right|<\delta\right\}
$$

with a $C^{0,1}$ norm bound

$$
|u|_{C^{0,1}(\bar{\Omega})} \leq C_{1}
$$

where $\rho_{1}, \rho_{2}$ are smooth functions since $\partial M$ is smooth and transversal to $\overline{q_{1} q_{2}}$. Note that $u$ is a viscosity solution of the equation

$$
G\left(D^{2} u, D u\right)=K \text { in } \bar{\Omega} .
$$

Let $\varphi$ be a smooth function defined on $\partial B_{r}$, where $B_{r} \subset \Omega_{0}$ is the $n$-ball of radius $r \leq \delta$ centered at the origin, satisfying $\varphi(0, \pm r)=0$ and

$$
\varphi\left(x^{\prime}, x_{n}\right) \geq \max \left\{u\left(x^{\prime}, \rho_{1}\left(x^{\prime}\right)\right), u\left(x^{\prime}, \rho_{2}\left(x^{\prime}\right)\right)\right\}, \quad \forall\left(x^{\prime}, x_{n}\right) \in \partial B_{r} .
$$

This is possible since both $u\left(x^{\prime}, \rho_{1}\left(x^{\prime}\right)\right)$ and $u\left(x^{\prime}, \rho_{2}\left(x^{\prime}\right)\right)$ are smooth in $x^{\prime}$ as $\partial M$ is smooth and tangential to $x_{n+1}=0$.

Consider the Dirichlet problem

$$
F\left(D^{2} v\right) \equiv G\left(D^{2} v, 0\right)=K \text { in } \overline{B_{r}}, v=\varphi \text { on } \partial B_{r} .
$$

Under the assumptions (1.3)-(1.6) and (1.8), it follows from Theorem $2^{\prime}$ of [3] (see also [23]) that there exists a unique strictly convex solution $v \in C^{\infty}\left(\overline{B_{r}}\right)$ of $(4.2)$; this is the only place we need assumption (1.8).

By the convexity of $u$ we have $F\left(D^{2} u\right) \geq F(A[u])=K$ in $\overline{B_{r}}$ from $(2.38)$ and

$$
u\left(x^{\prime}, x_{n}\right) \leq \max \left\{u\left(x^{\prime}, \varphi_{1}\left(x^{\prime}\right)\right), u\left(x^{\prime}, \varphi_{2}\left(x^{\prime}\right)\right)\right\}, \quad \forall\left(x^{\prime}, x_{n}\right) \in \Omega_{0}
$$

which implies $v \geq u$ on $\partial B_{r}$. Therefore $v \geq u \geq 0$ on $\overline{B_{r}}$ by the comparison principle. On the other hand, we have $v(0)<0$ since $v(0, a)=v(0,-a)=0$ and $v$ is strictly convex. This is a contradiction.

We are now ready to prove the interior smoothness of $M$. Let $q \in M$ be an interior point which we may assume to be the origin of $\mathbb{R}^{n+1}$. Since $M$ is locally Lipschitz, $M$ locally near the origin can be represented as a convex graph $x_{n+1}=u(x) \geq 0$ over a domain $\Omega_{1} \subset \mathbb{R}^{n} \equiv\left\{x_{n+1}=0\right\}$ with a $C^{0,1}$ norm bound

$$
|u|_{C^{0,1}\left(\overline{\Omega_{1}}\right)} \leq C_{1} .
$$


By Lemma 4.1 we have $\{u \leq t\} \cap \partial \Omega_{1}=\emptyset$ for $t>0$ sufficiently small. It therefore follows from Theorem 2.9 that $u$ is smooth in $\{u<t\}$. Consequently, $M$ is smooth and strictly convex at $q$.

Finally, by an approximation argument the smoothness and strict convexity of $M$ at boundary points follow from the boundary estimates in section 2, combined with the $C^{2, \alpha}$ boundary estimates of Krylov [17] and the classical Schauder theory.

The proof of Theorem $1.1 \mathrm{c}$ ) is complete.

Remark 4.2. With some slight modifications one can use the above argument to prove the following interior smoothness when the boundary is not necessary smooth but is contained in a hyperplane, which has been used in the previous section to prove Theorem 1.3.

Lemma 4.3. Let $M$ be a locally convex hypersurface satisfying $f(\kappa[M]) \equiv K>0$ with $\partial M$ contained in a hyperplane, where $f$ satisfies the structure conditions (1.3)-(1.6). Then $M$ is smooth and locally strictly convex in interior.

\section{REFERENCES}

[1] S. Alexander and M. Ghomi, The convex hull property and the topology of hypersurfaces with nonnegative curvature, Adv. in Math., to appear.

[2] L. A. Caffarelli, L. Nirenberg and J. Spruck, The Dirichlet problem for nonlinear second-order elliptic equations I. Monge-Ampère equations, Comm. Pure Applied Math. 37 (1984), 369-402.

[3] L. A. Caffarelli, L. Nirenberg and J. Spruck, The Dirichlet problem for nonlinear second-order elliptic equations III: Functions of eigenvalues of the Hessians, Acta Math. 155 (1985), 261-301.

[4] L. A. Caffarelli, L. Nirenberg and J. Spruck, Nonlinear second order elliptic equations IV. Starshaped compact Weingarten hypersurfaces, Current Topics in P.D.E. Kinokunize Co., Tokyo 1986, 1-26 (Y. Ohya, et al. editors).

[5] L. A. Caffarelli, L. Nirenberg and J. Spruck, Nonlinear second order elliptic equations V. The Dirichlet problem for Weingarten hypersurfaces, Comm. Pure Applied Math. 41 (1988), 41-70.

[6] L. C. Evans, Classical solutions of fully nonlinear, convex, second order elliptic equations, Comm. Pure Applied Math. 35 (1982), 333-363.

[7] B. Guan, The Dirichlet problem for Monge-Ampère equations in non-convex domains and spacelike hypersurfaces of constant Gauss curvature, Trans. Amer. Math. Soc. 350 (1998), 4955-4971.

[8] B. Guan, The Dirichlet problem for Hessian equations on Riemannian manifolds, Calc. Var. 8 (1999), 45-69.

[9] B. Guan and P.-F. Guan, private communication, 1999.

[10] B. Guan and J. Spruck, Interior gradient estimates for solutions of prescribed curvature equations of parabolic type, Indiana Univ. Math. J. 40 (1991), 1471-1481.

[11] B. Guan and J. Spruck, Boundary value problem on $S^{n}$ for surfaces of constant Gauss curvature, Ann. of Math. 138 (1993), 601-624. 
[12] B. Guan and J. Spruck, The existence of hypersurfaces of constant Gauss curvature with prescribed boundary, J. Differential Geometry 62 (2002), 259-287.

[13] N. M. Ivochkina, Solution of the Dirichlet problem for curvature equations of order $m$, Math. USSR Sb. 67 (1990), 317-339.

[14] N. M. Ivochkina, The Dirichlet problem for the equations of curvature of order $m$, Leningrad Math. J. 2 (1991), 631-654.

[15] N. M. Ivochkina, M. Lin and N. S. Trudinger, The Dirichlet problem for the prescribed curvature quotient equations with general boundary values, Geometric Analysis and the Calculus of Variations, 125-141, Internat. Press, Cambridge, MA, 1996.

[16] N. M. Ivochkina and F. Tomi, Locally convex hypersurfaces of prescribed curvature and boundary, Calc. Var. 7 (1998), 293-314.

[17] N. V. Krylov, Boundedly nonhomogeneous elliptic and parabolic equations in a domain, Izv. Akad. Nauk. SSSR Ser. Mat. 47 (1983), 75-108 (Russian); English translation in Math. USSR Izv. 22 (1984), 67-97.

[18] M. Lin and N. S. Trudinger, The Dirichlet problem for the prescribed curvature quotient equations, Top. Methods in Nonlinear Analysis 3 (1994), 307-323.

[19] H. Rosenberg, Hypersurfaces of constant curvature in space forms, Bull. Sciences Mathematiques 117 (1993), 211-239.

[20] W.-M. Sheng, J. Urbas and X.-J. Wang, Interior curvature bounds for a class of curvature equations, Duke J. Math., to appear.

[21] J. Spruck, Geometric Aspects of the theory of fully nonlinear elliptic equations, Clay Mathematics Proceedings Vol. 3, (2003).

[22] N. S. Trudinger, The Dirichlet problem for the prescribed curvature equations, Arch. Rational Mech. Anal. 111 (1990), 153-179.

[23] N. S. Trudinger, On the Dirichlet problem for Hessian equations, Acta Math. 175 (1995), 151164.

[24] N. S. Trudinger and X.-J. Wang, On locally convex hypersurfaces with boundary, J. Reine Angew. Math. 551 (2002), 11-32.

Department of Mathematics, University of Tennessee, Knoxville, TN 37996, Fax: (865) 974-6576.

E-mail address: guan@math.utk.edu

Department of Mathematics, Johns Hopkins University, Baltimore, MD 21218, Fax: (410) 516-5549.

E-mail address: js@math.jhu.edu 